BMJ Open

Sport \&

Exercise

Medicine

\title{
Achilles tendinopathy - do plantaris tendon removal and Achilles tendon scraping improve tendon structure? A prospective study using ultrasound tissue characterisation
}

\author{
Lorenzo Masci, ${ }^{1}$ Christoph Spang, ${ }^{2}$ Hans T M van Schie, ${ }^{3}$ Håkan Alfredson ${ }^{1,4,5}$
}

To cite: Masci L, Spang C, van Schie HTM, et al. Achilles tendinopathy-do plantaris tendon removal and Achilles tendon scraping improve tendon structure?

A prospective study using ultrasound tissue characterisation. BMJ Open Sport Exerc Med 2015;1: e000005. doi:10.1136/ bmjsem-2015-000005

- Prepublication history for this paper is available online. To view these files please visit the journal online (http://dx.doi.org/10.1136/ bmjsem-2015-000005).

Received 2 March 2015 Revised 13 March 2015 Accepted 14 March 2015

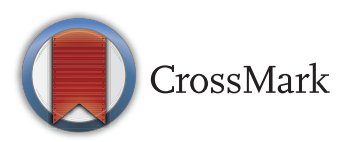

\footnotetext{
${ }^{1}$ Pure Sports Medicine Clinic, London, UK

${ }^{2}$ Department of Integrative Medical Biology (IMB), Anatomy Section, Umea University, Umeå, Sweden ${ }^{3}$ Department of Scientific Research, UTC Imaging, Stein, The Netherlands ${ }^{4}$ Department of Community Medicine and Rehabilitation, Umeå University, Sweden ${ }^{5}$ ISEH, University College London Hospitals, London, UK
}

Correspondence to Professor Håkan Alfredson; hakan.alfredson@umu.se

\section{ABSTRACT}

Objectives: The plantaris tendon has recently been described as a possible important factor in midportion Achilles tendinopathy. Ultrasound tissue characterisation (UTC) is a method to study tendon structure (matrix integrity). The effect of plantaris tendon removal on Achilles tendon structure was studied using UTC.

Design and setting: Prospective case series study at one centre.

Participants: Nine tendons in eight physically active and healthy patients (mean age 39 years) with chronic painful midportion Achilles tendinopathy were included. Preoperative two-dimensional ultrasound and UTC showed midportion Achilles tendinopathy (tendinosis) with medial tendon changes and suspected plantaris tendon involvement. Patients with previous operations to the Achilles tendon were excluded.

Interventions: Operative treatment consisted of excision of the plantaris tendon and scraping of the ventromedial surface of the Achilles tendon under a local anaesthetic.

Primary and secondary outcome measures: UTC examination and clinical scoring with the VISA-A questionnaire were performed preoperatively and 6 months postoperatively.

Results: At 6 months follow-up, UTC demonstrated a statistically significant $(t=5.40, p<0.001)$ increase in the mean organised matrix (echo-type $\mathrm{I}+\mathrm{II}$ ) and a decrease in the mean disorganised matrix (echo-type III+IV). Seven out of eight patients were satisfied, and the VISAA score had increased significantly $(p<0.001)$ from 56.8 (range 34-73) preoperatively to 93.3 (range 87-100) postoperatively.

Conclusions: Excision of the plantaris tendon and scraping of the ventromedial Achilles tendon in chronic midportion tendinopathy seem to have the potential to improve tendon structure and reduce tendon pain.

Studies on a larger group of patients and with a longer follow-up period are needed.

\section{INTRODUCTION}

Midportion Achilles tendinopathy is a condition that often presents with pain and disability
What are the new findings?

- Excision of the plantaris tendon and scraping of the ventromedial Achilles tendon in chronic midportion Achilles tendinopathy have the potential to improve Achilles tendon structure.

- Ultrasound tissue characterisation appears to be a promising tool to study tendon matrix changes, and can be useful to monitor healing processes.

- Excision of the plantaris tendon and scraping of the ventro-medial Achilles tendon in chronic midportion Achilles tendinopathy have the potential to reduce tendon pain.

during Achilles tendon loading activities. Despite extensive research, the pathogenesis is still not fully understood. ${ }^{1}{ }^{2}$ Treatment has been difficult to formalise, ${ }^{3}$ but recent innovative methods applied outside the tendon have shown promising results. ${ }^{4}$ A novel treatment method targeting the peritendinous tissues on the ventral side of the Achilles tendon, that is, the recently described minimally-invasive operative scraping procedure ${ }^{5}$ has resulted in a high success rate at 1-2-year follow-up. Nevertheless, not all patients are cured, and in some cases medial tendon pain has been shown to be related to the plantaris tendon. ${ }^{6} 7$

Ultrasound with colour Doppler (US+CD) has been used for many years as a first-line diagnostic tool to detect tendinosis-like changes in tendons, ${ }^{8} 9$ and a few studies used ultrasound to visualise the plantaris tendon. ${ }^{10}{ }^{11}$ Recently, a novel imaging modality called ultrasound tissue characterisation (UTC) was used to visualise Achilles tendon structure and to quantify tendon matrix integrity. ${ }^{12}$ Unlike two-dimensional (2D) US and CD, UTC objectively quantifies grey-scale tendon matrix changes into four different echotypes related to tendon integrity. Type I (green) and II (blue) represent 
organised matrix; type III (red) and IV (black) represent a disorganised matrix.

Clinically, we have had good results, with high patient satisfaction and a fast return to Achilles tendon loading activities, after removal of the plantaris tendon and surgical scraping of the Achilles tendon. ${ }^{13}$ Our hypothesis is that removing the plantaris tendon and scraping the Achilles tendon in patients with chronic painful midportion Achilles tendinopathy results in improved tendon structure.

The aim of this study was to prospectively investigate the outcome of plantaris tendon excision and operative scraping in patients with midportion Achilles tendinopathy (tendinosis) and suspected plantaris tendon involvement, by using UTC to study the tendon structure and the VISA-A score for clinical outcome.

\section{METHODS}

A prospective study design was implemented using UTC to study tendon structure and the VISA-A score to study clinical results after surgical treatment of patients with chronic painful midportion Achilles tendinopathy.

All examinations, before and after surgery, were performed at the Pure Sports Medicine Clinic in London, UK, between August 2013 and August 2014.

The included patients were referrals from general practitioners, physiotherapists and sports medicine physicians within the UK.

\section{Participants}

Patients with midportion Achilles tendinopathy with medial tendon pain (history), medial tenderness (palpation) and localised medial tendon abnormalities (US and UTC), were included in the study.

Nine Achilles tendons from 8 patients $(7$ men and 1 woman) with a mean age of 39 years (range $26-56$ years) were included. Duration of symptoms ranged from 6 to 48 months. All patients complained of activity-related medial tendon pain and had tenderness localised to the medial side of the Achilles tendon. All patients had failed a tendon loading programme (eccentric training). All patients were physically active, consisting of six runners (two elite level), one cricket player and one footballer.

\section{Presurgical examinations}

Clinical assessment

Patients were asked to determine the site of activityrelated tendon pain. Patients were examined in the prone position with feet hanging freely over the edge of the examination bed. Tendons were assessed by the same examiner (HA) for thickening and location of tenderness. All patients included in this study had medial tenderness.

\section{US and CD examination}

Patients were examined in prone position with feet hanging freely over the edge of the examination bed.
High-resolution greyscale Ultrasound (US) and colour Doppler (CD) (Antares-Siemens), with a linear multifrequency $(8-13 \mathrm{MHz})$ probe, were used to evaluate tendon thickness, structure and blood flow. CD settings were standardised with a gain of $68 \mathrm{~dB}$, sensitivity of $8 \mathrm{~cm} / \mathrm{s}$ and a pulse repetition frequency of $1250 \mathrm{~Hz}$. Scans were performed by an experienced practitioner in ultrasound diagnostics (HA). Achilles tendon structure was evaluated as either normal, or irregular and hypoechoic. The location of the hypoechogenicity was noted (medial, ventral, lateral). Achilles tendons were characterised as having normal or high blood flow. Normal flow tendons had no detectable blood flow in the sagittal or axial plane, whereas high-blood flow tendons contained at least one region with high-blood flow in the sagittal or axial plane. The location of the blood flow was noted (medial or lateral). Achilles tendinopathy (tendinosis) was defined as a thickened tendon midportion with irregular structure, hypoechogenicity and localised blood flow. In addition, scans were analysed for the presence of a plantaris tendon located close to the medial side of the Achilles tendon midportion. Tendons included in this study exhibited medial hypoechogenicity and medial blood flow.

\section{Ultrasound tissue characterisation}

UTC (UTC Imaging, Stein, the Netherlands) was used to tomographically visualise the Achilles tendon. ${ }^{10}$ A high-resolution 7-12 MHz linear US probe (Teratech, USA) was fixed to a tracking device that moved the transducer automatically along the tendon. In this way, contiguous transverse US images were collected at even distances of $0.2 \mathrm{~mm}$ over a distance of $12 \mathrm{~cm}$.

Patients were positioned prone on the examination table with the affected ankle caudal to the edge of the examination bed. The examiner's knee was pushed against the patient's forefoot to achieve maximal passive dorsiflexion (figure 1). Coupling gel was applied to the skin, and between the probe and stand off, to optimise sound wave penetration. The tracking device was placed on the posterior aspect of the lower leg and parallel to the longitudinal axis of the Achilles tendon with the caudal edge of the standoff in line with the caudal aspect of the heel. Scans were collected in a distal to proximal direction and were all performed by the same experienced examiner (LM). UTC has shown a high intra-rater and inter-rater reliability. ${ }^{12} 14$

Images were stored real-time in a high-capacity laptop computer and subsequent compounding of these images created a three-dimensional (3D) volume block of US data. The 3D data block was used for tomographical reconstructions (transverse, sagittal, coronal and 3D coronal planes of view). Dedicated UTC algorithms (UTC 2010, UTC Imaging) were used to quantify the dynamics of grey levels of corresponding pixels in contiguous images over 25 images. Previous fundamental research has shown that the dynamics of grey levels is strongly related to the architecture and integrity of the 


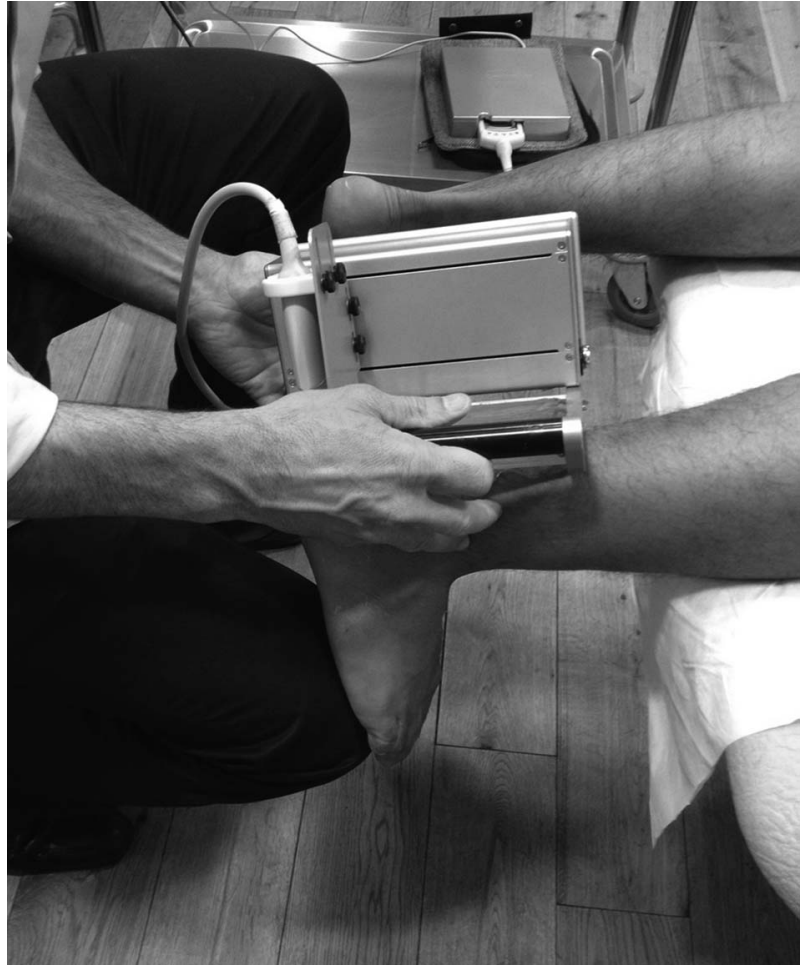

Figure 1 Examination using ultrasound tissue characterisation (UTC). The patient is positioned prone on an examination table with the affected ankle caudal to the edge of the examination bed. The examiner's knee is pushed against the patient's forefoot to achieve maximal dorsiflexion.

tendon matrix with histomorphology of tissue specimen as reference test. ${ }^{15-18}$ In this way, validated UTC algorithms can discriminate four different echotypes, related to matrix integrity:

- Type I is generated by intact, continuous and aligned collagen bundles (fibres and fasciculi).

- Type II is generated by less continuous and/or more swollen and/or more wavy collagen bundles (fibres and fasciculi).

- Type III represents some disintegration with tendon tissue replaced by a mainly disorganised, fibrillar matrix.

- Type IV represent complete disintegration with tendon tissue replaced by an amorphous matrix and fluid.

For each examined tendon, the percentage of each variety of echo-types was taken before and after treatment (follow-up; see below). Once quantified, scans were analysed by the experienced examiner (LM) for regions with disintegration (echopixel types III and IV) within the midportion of the Achilles tendon and recorded according to site (superficial, ventral, medial or lateral). Achilles tendons of the included patients showed disintegration primarily in the medial aspect.

\section{Operation}

\section{Surgery}

Surgery was performed within $24 \mathrm{~h}$ after UTC, US+CD and clinical assessment. During surgical treatment under local anaesthesia, ${ }^{6}$ the medial aspect of the tendon was visualised via a short longitudinal incision on the medial side of the Achilles tendon midportion (figure 2A). A careful inspection of the medial side of the Achilles tendon was performed by the surgeon (HA) to determine the presence and exact location of a plantaris tendon. When detected, the location in relation to the medial aspect of the Achilles tendon was documented with photographs. In all patients, a thickened plantaris tendon was found in close relationship to the medial aspect of the Achilles tendon, seemingly compressing it. The surgical treatment consisted of release of the plantaris tendon followed by excision of the plantaris tendon distally from the calcaneal insertion and proximally at a level slightly above the distal medial soleus muscle insertion (figure 2B). In addition, a tendon scraping procedure was performed in the regions demonstrating Ultrasound+Doppler-verified highblood flow on the ventromedial side of the Achilles tendon (figure 3). ${ }^{5}$ Here, the tendon was released from the ventromedial soft tissue by sharp dissection with a knife.

\section{Follow-up}

A follow-up was performed 6 months after surgical treatment. Tendon structure was analysed with UTC and VISA-A score was taken. Patient satisfaction with the result of the operation was evaluated by self-reporting (satisfied or not satisfied). The outcome was considered to be 'good' when patients were satisfied with the treatment and when they were back to full Achilles tendon loading.
Figure 2 Surgical excision of the plantaris tendon. Visualisation (A), and release and removal of the plantaris tendon (B) close to the medial side of the Achilles tendon.
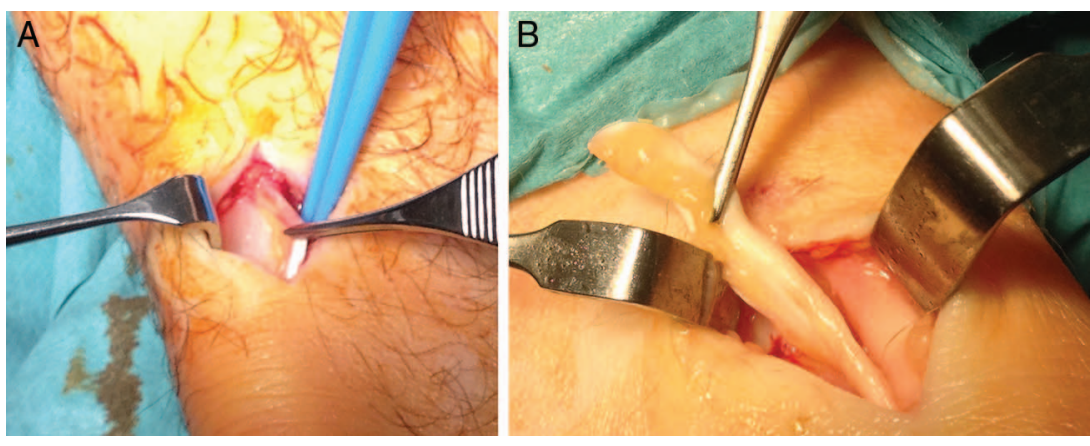


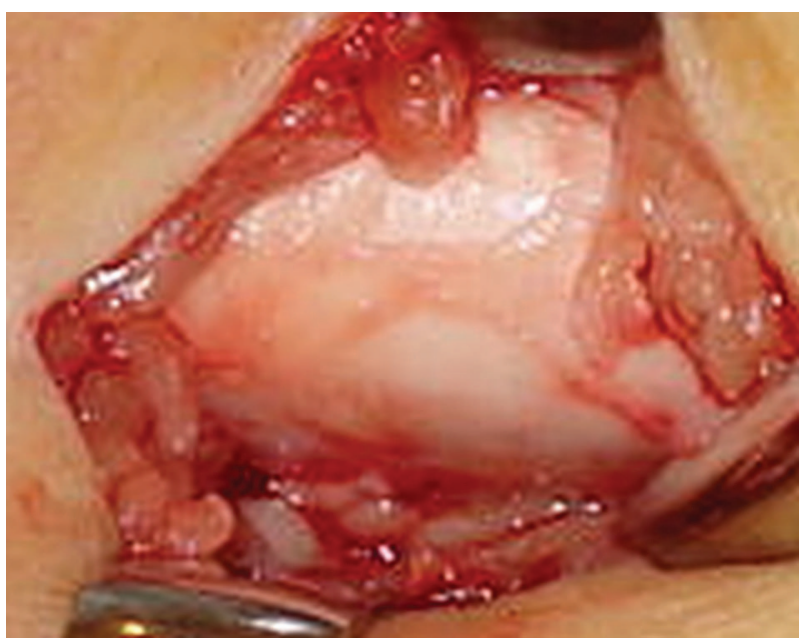

Figure 3 Scraping the ventromedial side of the Achilles tendon. The infiltrative fat tissue on the ventromedial side of the Achilles tendon has been scraped loose.

\section{Statistics}

SPSS (Statistical Package of Social Science) was used to analyse the data (SPSS Inc, Chicago, Illinois, USA). Parametric tests ( $t$ test) were performed, where level of significance was set to $\mathrm{p}<0.05$.

\section{Ethics}

The study was approved by University of Queen Mary ethics committee as part of a prospective study on all surgically-treated Achilles tendons at Pure Sports Medicine Clinic in London, UK. All participants provided written informed consent prior to participation.

\section{RESULTS}

All patients (tendons) participated in all preclinical examinations and the 6 months follow-up.

At the 6 months follow-up, UTC demonstrated a statistically significant $(\mathrm{p}<0.001)$ increase in the mean number (in percent) of organised echopixels (echo-type I+II) from 83.33 (range 74-95) to 91.33 (76-97; figure 4 ). The mean number (in percent) of disorganised echopixels (echo-type III+IV) decreased from 16.66 (range 5.1-29.9) to 8.67 (range 3.6-14.2; figure 4$)$. These findings were consistent in all patients.

Seven out of eight patients (8/9 tendons) were satisfied with the result of the treatment.

The VISA-A score had significantly increased $(\mathrm{p}<0.001)$ from 56.8 (range 34-73) preoperatively to 93.3 (range $87-100$ ) postoperatively (figure 4).

\section{DISCUSSION}

This study showed that, in a group of patients with midportion Achilles tendinopathy (having medial tendon pain and tenderness), there was significant improvement in their symptoms and in the structure of the Achilles tendon, 6 months after surgical removal of the plantaris

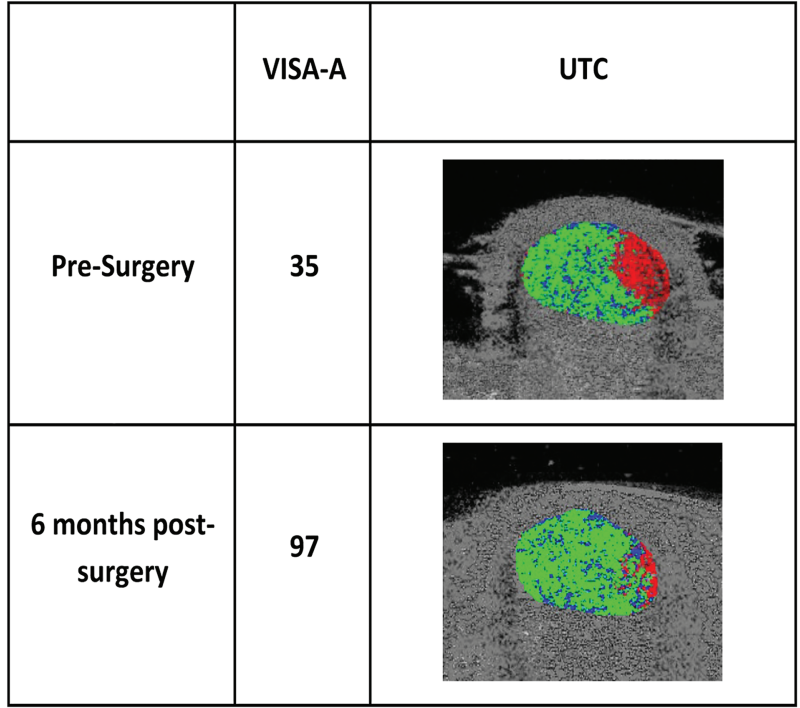

Figure 4 Ultrasound tissue characterisation (UTC) findings and VISA-A scores. VISA-A score and UTC findings before and 6 months after surgery in a patient with midportion Achilles tendinopathy and plantaris tendon involvement. Note the medially located changes (red pixels) on UTC.

tendon and surgical scraping of the ventromedial side of the Achilles tendon. Clinically, the results were good, with significantly improved VISA-A scores and seven out of the eight patients were satisfied with the result.

A limitation of the study was the relatively small sample size, but the UTC findings were consistent across all patients. Another limitation is that the UTC methodology is relatively new and, although there is good experience from using UTC on horse flexor tendons, there is less experience with human tendons.

From this study, we cannot differentiate whether the positive effects on the tendon structure and the good clinical results were achieved by the removal of the plantaris tendon or the scraping procedure of the ventromedial Achilles tendon. However, the macroscopic findings at operation showed compression from the thick plantaris tendon on the medial side of the Achilles tendon. Furthermore, preoperative UTC scans showed disintegration primarily in the ventromedial aspect of the Achilles tendon where the plantaris tendon touches the Achilles tendon. This was accompanied by medial pain and medial tenderness. We have postulated that the closely located plantaris tendon might have compressed the medial Achilles tendon. ${ }^{6}$ The compression theory, raised by Cook and Purdam for insertional tendinopathies, ${ }^{19}$ might also apply to midportion Achilles tendinopathy. It is well known that the plantaris tendon is stronger and stiffer than the Achilles tendon, ${ }^{20}$ and a position close to, and sometimes grooving the medial side of the Achilles tendon, might possibly cause compressive and/or shearing forces. This theory is further supported by the fact that the plantaris muscle/tendon complex produces both ankle flexion and inversion and could compress the Achilles tendon when in close 
apposition. ${ }^{21}$ The structural matrix changes in chronic painful Achilles tendinopathy are potentially reversible, and there would appear to be potential for remodelling of the Achilles tendon after removal of a compressing plantaris tendon.

The results of the current study suggest that there is a relationship between tendon structure (matrix integrity) and pain. There was an improved matrix integrity in conjunction with an improved clinical VISA-A score over time. Previous US studies have suggested that the tendon structure in tendinopathy is not related to pain symptoms. ${ }^{22}$ and this may need to be reconsidered in the light of more recent research using UTC.

We used UTC to study the tendon structure (matrix integrity), and this method has been shown to be reliable and valid, with a high intra-rater and interrater reliability. ${ }^{10}{ }^{12}$ UTC is a new method to study tendon structure (matrix integrity) that allows for quantification, whereas the traditionally used 2D US does not. Previous UTC studies have demonstrated statistical differences in percentage of echotypes between symptomatic tendinopathy tendons and asymptomatic normal Achilles tendons. ${ }^{10}$ Asymptomatic tendons had significantly higher percentages of type I and II echopixels, indicative of an organised matrix, whereas symptomatic tendons had significantly higher type III and IV echopixels, indicative of a disorganised tendon matrix.

In conclusion, by using UTC to follow the tendon structure (matrix integrity) after plantaris tendon removal and surgical scraping in patients with chronic painful midportion Achilles tendinopathy, it was shown that there was an improvement of the Achilles tendon structure, in parallel with improved clinical VISA-A scores. UTC seems to be a promising tool to study tendon matrix changes, and can potentially be useful to evaluate Achilles tendons and monitor healing.

Contributors LM performed all clinical and UTC examinations and was in charge of all protocols and files. He was involved in the evaluation of the results and also participated in manuscript writing. HA performed all clinical and Ultrasound+Doppler examinations and operations. He also participated in manuscript writing. CS was involved in the collection and evaluation of the results, and participated in manuscript writing. HTMvS controlled and double checked all UTC examinations and participated in manuscript writing.

Competing interests HTMvS is the inventor of the UTC device. He has not given any financial support for this study and has no financial interests in relation to this study.

Ethics approval University of Queen Mary ethics committee.

Provenance and peer review Not commissioned; externally peer reviewed.

Open Access This is an Open Access article distributed in accordance with the Creative Commons Attribution Non Commercial (CC BY-NC 4.0) license, which permits others to distribute, remix, adapt, build upon this work noncommercially, and license their derivative works on different terms, provided the original work is properly cited and the use is non-commercial. See: http:// creativecommons.org/licenses/by-nc/4.0/

\section{REFERENCES}

1. Fu SC, Rolf C, Cheuk YC, et al. Deciphering the pathogenesis of tendinopathy: a three stage process. Sports Med Arthrosc Rehabil Ther Techonol 2010;2:30.

2. Cook JL, Purdham CR. Is tendon pathology a continuum? A pathology model to explain the clinical presentation of load-induced tendinoapthy. Br J Sports Med 2009;43:409-16.

3. Cook JL, Purdam CR. The challenge of managing tendinopathy in competing athletes. Br J Sports Med 2014;48:506-9.

4. Alfredson $\mathrm{H}$, Ohberg $\mathrm{L}$, Zeisig $\mathrm{E}$, et al. Treatment of midportion Achilles tendinosis: similar clinical results with US and CD-guided surgery outside the tendon and sclerosing polidocanol injections. Knee Surg Sports Traumatol Arthrosc 2007;15:1504-9.

5. Alfredson $\mathrm{H}$. Ultrasound and Doppler-guided mini-surgery to treat mid-portion Achilles tendinosis: results of a large material and a randomised study comparing two scraping techniques. $\mathrm{Br} J$ Sports Med 2011;45:407-10.

6. Alfredson $\mathrm{H}$. Mid-portion Achilles tendinosis and the plantaris tendon. Br J Sports Med 2011;45:1023-5.

7. Spang $\mathrm{C}$, Alfredson $\mathrm{H}$, Ferguson $\mathrm{M}$, et al. The plantaris tendon in association with mid-portion Achilles tendinosis: tendinosis-like morphological features and presence of a non-neuronal cholinergic system. Histol Histopathol 2013;28:623-32.

8. Ohberg L, Lorentzon R, Alfredson H. Neovascularisation in Achilles tendons with painful tendinosis but not in normal tendons: an ultrasonographic investigation. Knee Surg Sports Traumatol Arthrosc 2001;9:233-8.

9. Mitchell AW, Lee JC, Healy JC. The use of ultrasound in the assessment and treatment of Achilles tendinosis. J Bone Joint Surg Br 2009;91:1405-9.

10. Mackay IR, McCulloch AS. Imaging the plantaris tendon with ultrasound. Br J Plast Surg 1990;43:689-91.

11. Spina AA. The plantaris muscle: anatomy, injury, imaging, and treatment. J Can Chiropr Assoc 2007;51:158-65.

12. Van Schie HT, de Vos RJ, de Jonge S, et al. Ultrasonographic tissue characterisation of human Achilles tendons: quantification of tendon structure through a novel non-invasive approach. $\mathrm{Br} J$ Sports Med 2010;44:1153-9.

13. Ruergard A, Alfredson H. Major physical but also psychological effects after pain relief from surgical scraping in patients with Achilles tendinopathy-a 1-year follow-up study. Pain Stud Treat 2014;2:21-5.

14. Rosengarten SD, Cook JL, Bryant AL, et al. Australian football players' Achilles tendons respond to game loads within 2 days: an ultrasound tissue characterisation (UTC) study. Br J Sports Med 2015;49:183-7.

15. van Schie HT, Bakker EM. Structure-related echoes in ultrasonographic images of equine superficial digital flexor tendons. Am J Vet Res 2000;61:202-9.

16. van Schie HT, Bakker EM, Jonker AM, et al. Efficacy of computerized discrimination between structure-related and non-structure-related echoes in ultrasonographic images for the quantitative evaluation of the structural integrity of superficial digital flexor tendons in horses. Am J Vet Res 2001;62:1159-66.

17. van Schie HT, Bakker EM, Jonker AM, et al. Computerized ultrasonographic tissue characterization of equine superficial digital flexor tendons by means of stability quantification of echo patterns in contiguous transverse ultrasonographic images. Am J Vet Res 2003;64:366-75.

18. Van Schie HT, Bakker EM, Cherdchutham W, et al. Monitoring of the repair process of surgcially created lesions in equine superficial digital flexor tendons by use of computerized ultrasonography. $A m \mathrm{~J}$ Vet Res 2009;70:37-48.

19. Cook JL, Purdam C. Is compressive load a factor in the development of tendinopathy? Br J Sports Med 2012;46:163-8.

20. Lintz $F$, Higgs $A$, Millett $M$, et al. The role of plantaris longus in Achilles tendinopathy: a biomechanical study. J Foot Ankle Surg 2011;17:252-5.

21. van Sterkenburg MN, van Dijk CN. Mid-portion Achilles tendinopathy: why painful? An evidence-based philosophy. Knee Surg Sports Traumatol Arthrosc 2011;19:1367-75.

22. Khan KM, Cook JL, Bonar F, et al. Histopathology of common tendinopathies. Update and implications for clinical management. Sports Med 1999;27:393-408. 